\title{
Chemical modifications of vicilins interfere with chitin-binding affinity and toxicity to Callosobruchus maculatus (Coleoptera: Chrysomelidae) insect: a combined in vitro and in silico analysis
}

\author{
Maria Raquel A. Miranda ${ }^{a}$, Adriana F. Uchôa ${ }^{b}$, Sarah R. Ferreirac ${ }^{c}$ Kayan E. \\ Venturyc ${ }^{c}$ Evenilton P. Costac, Paulo R. Leitão Carmo ${ }^{d}$, Olga L.T. Machadoc, \\ Katia V.S. Fernandes ${ }^{c}$ and Antonia Elenir A. Oliveirac*.
}

aDepartamento de Bioquímica, Centro de Ciências, Universidade Federal do Ceará-UFC, Fortaleza-Ce, Brazil.

bDepartamento de Biologia Celular e Genética, Centro de Biociências, Universidade Federal do Rio Grande do Norte, Natal-RN, Brazil.

cCentro de Biociências e Biotecnologia, Universidade Estadual do Norte Fluminense Darcy Ribeiro-UENF, Campos dos Goytacazes-RJ, Brazil. dNUPEN, Universidade Federal do Rio de Janeiro (UFRJ) Macaé -RJ, Brazil.

\section{AUTHOR INFORMATION}

\section{Corresponding Author}

Antonia Elenir Amancio Oliveira: Laboratorio de Quimica e Função de Proteínas e Peptídeos, Centro de Biociências e Biotecnologia, Universidade Estadual do Norte Fluminense Darcy Ribeiro-UENF, Campos dos Goytacazes-RJ, Brazil. ZIP POSTAL CODE: 28013-602. orcid.org/0000-0002-2618-8916. Tel 55 (22) 27397132. 
Supporting Information for Publication

Figures S1-4 and Table S1-3 


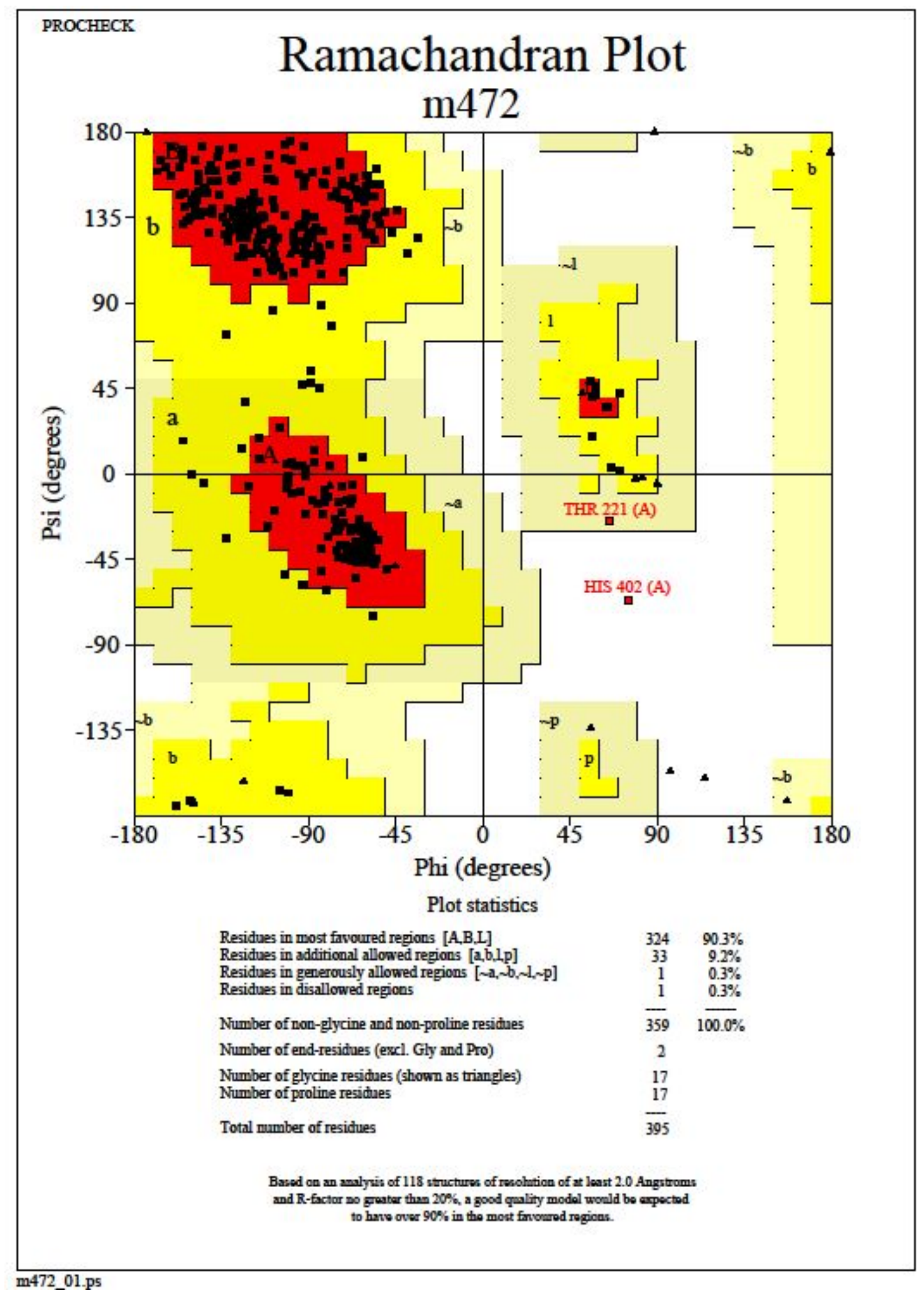

Figure S1: Stereochemical quality analyses by Ramachandran plot. The homology model predicted by Swiss-Model had its stereochemical quality analyzed by Procheck program on PDBsum. 


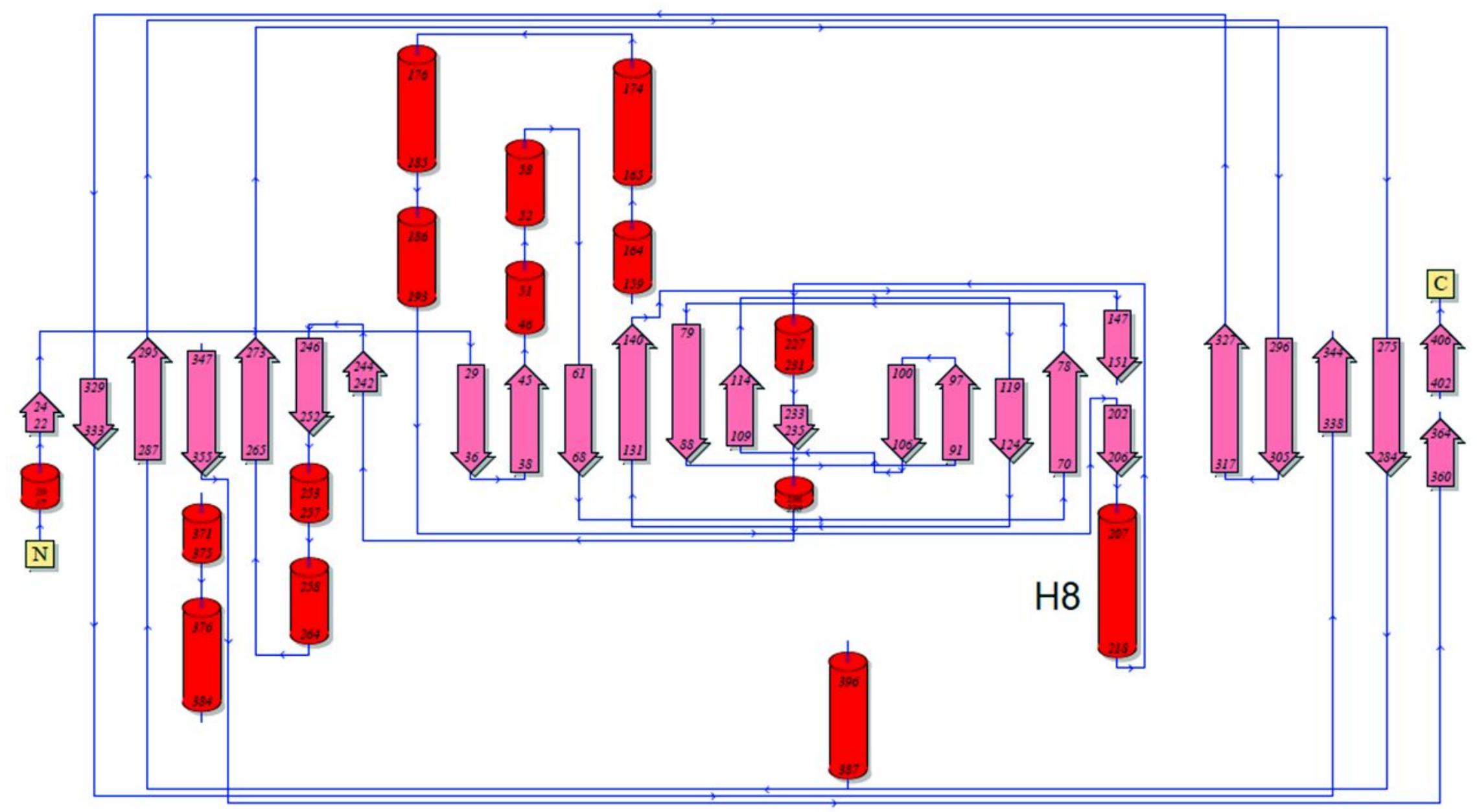

Figure S2: Vicilin structure topology. The vicilin topology was obtained by PDBsum program. The potential chitin-binding site located on alpha-helix 8 (H8) comprises the amino acids Arg208 up to Lys216. 

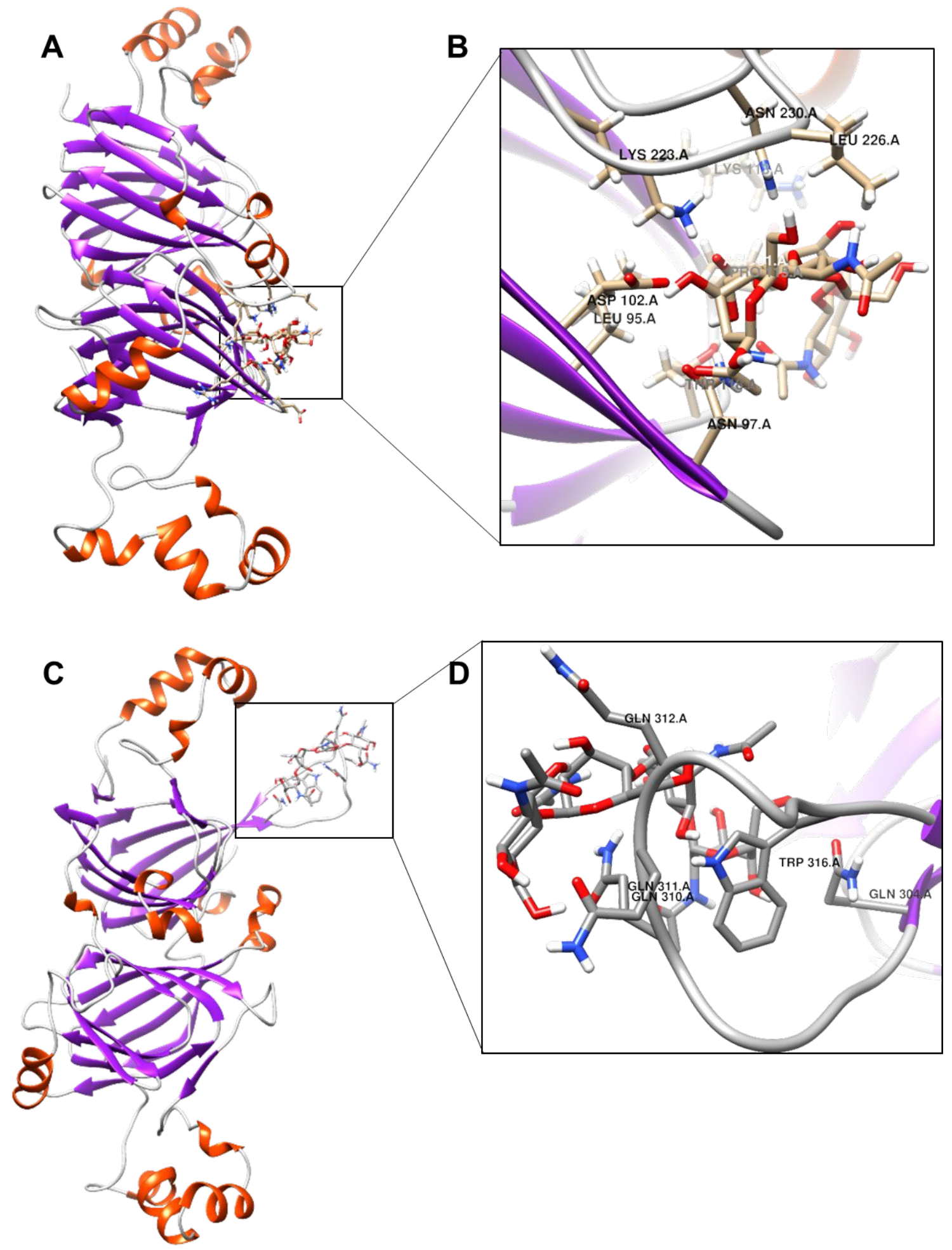

Figure S3- A and C: Vicilin-chitin complex after docking assays highlighting the position of the amino acid LYS223 (A) and TRP316 (C) in the structure. Loops and disordered regions in grey, alpha-helix in orange and beta-sheet in purple. $\mathbf{B}$ and $\mathbf{D}$ : Zoom of the binding site showing the amino acid LYS223 (B) and TRP316 (D) and the chitin in tan stick representation, nitrogen atoms in blue, oxygen atoms in red, hydrogen atoms in white, carbon atoms in tan, and side chains involved in the chitin-binding shown in stick representation. 
Table S1. Local and global quality assessment of vicilin structures.

\begin{tabular}{|c|c|c|c|c|c|c|c|c|c|c|c|}
\hline $\begin{array}{c}\text { Vicilin } \\
\text { predicted by }\end{array}$ & Length & $\begin{array}{c}\text { Verify3D } \\
(\%)\end{array}$ & $\begin{array}{c}\text { ProSa } \\
\text { Z-Score }\end{array}$ & VoroQMA & $\begin{array}{c}\text { ProQ } \\
\text { 2D } \\
\text { cad }\end{array}$ & $\begin{array}{c}\text { ProQ } \\
\text { 3D } \\
\text { cad }\end{array}$ & $\begin{array}{c}\text { ProQ } \\
\text { 2D } \\
\text { lddt }\end{array}$ & $\begin{array}{c}\text { ProQ } \\
\text { 3D } \\
\text { Iddt }\end{array}$ & DeepQA & Qprob & SVMQA \\
\hline RaptorX & 395 & 89.11 & -5.15 & 0.464 & 0.429 & 0.510 & 0.597 & 0.707 & 0.64971 & 0.580 & 0.8555 \\
\hline Swiss Model & $/ /$ & 93.92 & -6.52 & 0.500 & 0.441 & 0.538 & 0.621 & 0.742 & 0.71172 & 0.594 & 0.9008 \\
\hline $\begin{array}{c}\text { I-Tasser } \\
\text { model1 }\end{array}$ & $/ /$ & 94.68 & -6.23 & 0.486 & 0.435 & 0.500 & 0.603 & 0.678 & 0.68168 & 0.588 & 0.8226 \\
\hline model 2 & $/ /$ & 89.62 & -4.91 & 0.431 & 0.387 & 0.423 & 0.519 & 0.599 & 0.44305 & 0.540 & 0.7422 \\
\hline model 3 & $/ /$ & 95.44 & -6.19 & 0.487 & 0.430 & 0.475 & 0.613 & 0.670 & 0.62964 & 0.577 & 0.8201 \\
\hline model 4 & $/ /$ & 87.59 & -4.94 & 0.449 & 0.385 & 0.425 & 0.541 & 0.614 & 0.48706 & 0.543 & 0.7714 \\
\hline model 5 & $/ /$ & 84.05 & -3.90 & 0.417 & 0.393 & 0.441 & 0.544 & 0.617 & 0.42504 & 0.531 & 0.7257 \\
\hline
\end{tabular}

Cyan: $1^{\text {st }}$ place; Green: $2^{\text {nd }}$ place. 
Table S2. Accessible surface area and accessibility of the vicilin region (Arg208-Lys216) in monomer and homotrimer configuration.

\section{Vicilin Monomer}

Chain Residue $\begin{array}{cc}\text { Area } & \text { Relative } \\ \left(\AA^{2}\right) & (0.0-1.0)\end{array}$ Chain Residue

\begin{tabular}{|c|c|c|c|c|c|c|c|c|c|c|c|c|c|c|c|}
\hline A & Arg208 & 89.957 & 0.392 & $A$ & Arg208 & 139.026 & 0.606 & B & Arg208 & 138.148 & 0.602 & C & Arg208 & 136.627 & 0.595 \\
\hline A & Glu209 & 113.142 & 0.600 & A & Glu209 & 99.819 & 0.530 & B & Glu209 & 98.475 & 0.523 & C & Glu209 & 99.477 & 0.528 \\
\hline A & GIn210 & 81.693 & 0.437 & A & GIn210 & 49.392 & 0.264 & B & Gln210 & 48.691 & 0.261 & C & GIn210 & 48.798 & 0.261 \\
\hline A & Ile211 & 0.000 & 0.000 & $A$ & Ile211 & 4.199 & 0.021 & B & Ile211 & 4.426 & 0.023 & C & Ile211 & 4.086 & 0.021 \\
\hline A & Arg212 & 150.228 & 0.655 & A & Arg212 & 133.483 & 0.582 & B & Arg212 & 132.688 & 0.578 & C & Arg212 & 132.595 & 0.578 \\
\hline A & Glu213 & 86.041 & 0.457 & $A$ & Glu213 & 70.863 & 0.376 & B & Glu213 & 70.386 & 0.374 & C & Glu213 & 70.842 & 0.376 \\
\hline A & Leu214 & 40.820 & 0.198 & A & Leu214 & 3.765 & 0.018 & B & Leu214 & 3.878 & 0.019 & C & Leu214 & 3.692 & 0.018 \\
\hline A & Met215 & 30.627 & 0.141 & A & Met215 & 67.700 & 0.313 & B & Met215 & 67.686 & 0.312 & C & Met215 & 67.543 & 0.312 \\
\hline A & Lys216 & 156.545 & 0.732 & A & Lys216 & 132.301 & 0.619 & B & Lys216 & 132.466 & 0.620 & C & Lys216 & 132.950 & 0.622 \\
\hline
\end{tabular}

The parameters were obtained in: http://cib.cf.ocha.ac.jp/bitool/ASA/

\section{Vicilin Homotrimer}

Area Relative Chain Residue Area Relative Chain Residue Area Relative

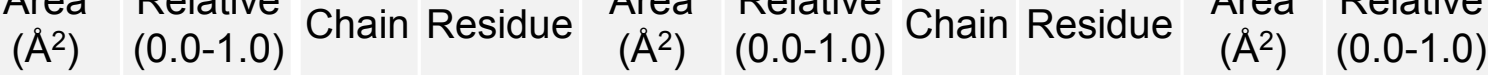


Table S3. Accessible surface area and accessibility of the residues of Tyrosine, Lysine and tryptophan in monomer and homotrimer configuration of vicilin. 


\begin{tabular}{|c|c|c|c|c|c|c|c|c|c|c|c|c|c|c|c|}
\hline \multicolumn{4}{|c|}{ Vicilin Monomer } & \multicolumn{12}{|c|}{ Vicilin Homotrimer } \\
\hline Chain & Residue & $\begin{array}{l}\text { Area } \\
\left(\AA^{2}\right)\end{array}$ & $\begin{array}{c}\text { Relative } \\
(0.0-1.0)\end{array}$ & Chain & Residue & $\begin{array}{l}\text { Area } \\
\left(\AA^{2}\right)\end{array}$ & $\begin{array}{c}\text { Relative } \\
(0.0-1.0)\end{array}$ & Chain & Residue & $\begin{array}{l}\text { Area } \\
\left(\AA^{2}\right)\end{array}$ & $\begin{array}{c}\text { Relative } \\
(0.0-1.0)\end{array}$ & Chain & Residue & $\begin{array}{l}\text { Area } \\
\left(\AA^{2}\right)\end{array}$ & $\begin{array}{c}\text { Relative } \\
(0.0-1.0)\end{array}$ \\
\hline A & Tyr38 & 64.300 & 0.266 & A & Tyr38 & 65.609 & 0.272 & B & Tyr38 & 66.050 & 0.273 & $\mathrm{C}$ & Tyr38 & 66.074 & 0.274 \\
\hline A & Tyr60 & 29.893 & 0.124 & A & Tyr60 & 30.959 & 0.128 & B & Tyr60 & 30.885 & 0.128 & $\mathrm{C}$ & Tyr60 & 30.701 & 0.127 \\
\hline A & Tyr104 & 1.311 & 0.005 & A & Tyr104 & 59.040 & 0.244 & B & Tyr104 & 57.906 & 0.240 & $\mathrm{C}$ & Tyr104 & 58.534 & 0.242 \\
\hline A & Tyr160 & 71.970 & 0.298 & A & Tyr160 & 2.736 & 0.011 & $\mathrm{~B}$ & Tyr160 & 2.772 & 0.011 & $\mathrm{C}$ & Tyr160 & 2.835 & 0.012 \\
\hline A & Tyr242 & 20.660 & 0.086 & A & Tyr242 & 13.311 & 0.055 & B & Tyr242 & 13.282 & 0.055 & $\mathrm{C}$ & Tyr242 & 13.188 & 0.055 \\
\hline A & Tyr282 & 52.261 & 0.216 & A & Tyr282 & 0.000 & 0.000 & B & Tyr282 & 0.000 & 0.000 & $\mathrm{C}$ & Tyr282 & 0.000 & 0.000 \\
\hline A & Tyr321 & 12.418 & 0.051 & A & Tyr321 & 6.932 & 0.029 & B & Tyr321 & 7.629 & 0.032 & $\mathrm{C}$ & Tyr321 & 9.080 & 0.038 \\
\hline A & Tyr337 & 50.302 & 0.208 & A & Tyr337 & 0.390 & 0.002 & B & Tyr337 & 0.231 & 0.001 & $\mathrm{C}$ & Tyr337 & 0.390 & 0.002 \\
\hline A & Lys52 & 149.148 & 0.698 & A & Lys52 & 84.097 & 0.393 & B & Lys52 & 85.118 & 0.398 & $\mathrm{C}$ & Lys52 & 84.695 & 0.396 \\
\hline A & Lys68 & 93.372 & 0.437 & A & Lys68 & 89.841 & 0.420 & B & Lys68 & 89.624 & 0.419 & $\mathrm{C}$ & Lys68 & 91.337 & 0.427 \\
\hline A & Lys 113 & 29.996 & 0.140 & A & Lys 113 & 13.698 & 0.064 & B & Lys 113 & 13.707 & 0.064 & $\mathrm{C}$ & Lys 113 & 13.630 & 0.064 \\
\hline A & Lys 135 & 6.151 & 0.029 & A & Lys 135 & 2.239 & 0.010 & B & Lys 135 & 2.178 & 0.010 & $\mathrm{C}$ & Lys 135 & 2.178 & 0.010 \\
\hline A & Lys 178 & 134.104 & 0.627 & A & Lys 178 & 145.773 & 0.682 & B & Lys 178 & 146.077 & 0.683 & $\mathrm{C}$ & Lys 178 & 146.309 & 0.685 \\
\hline A & Lys 191 & 159.855 & 0.748 & A & Lys 191 & 140.534 & 0.658 & B & Lys 191 & 141.128 & 0.660 & $\mathrm{C}$ & Lys 191 & 141.985 & 0.664 \\
\hline A & Lys207 & 142.877 & 0.668 & A & Lys207 & 140.318 & 0.656 & B & Lys207 & 139.998 & 0.655 & $\mathrm{C}$ & Lys207 & 139.957 & 0.655 \\
\hline A & Lys 216 & 156.545 & 0.732 & A & Lys 216 & 132.301 & 0.619 & B & Lys 216 & 132.466 & 0.620 & $\mathrm{C}$ & Lys 216 & 132.950 & 0.622 \\
\hline A & Lys219 & 65.438 & 0.306 & A & Lys219 & 154.576 & 0.723 & B & Lys219 & 153.318 & 0.717 & $\mathrm{C}$ & Lys219 & 154.638 & 0.723 \\
\hline A & Lys 223 & 22.605 & 0.106 & A & Lys 223 & 164.360 & 0.769 & B & Lys 223 & 165.154 & 0.773 & $\mathrm{C}$ & Lys 223 & 163.683 & 0.766 \\
\hline A & Lys224 & 153.177 & 0.717 & A & Lys224 & 176.122 & 0.824 & B & Lys224 & 176.163 & 0.824 & $\mathrm{C}$ & Lys224 & 174.465 & 0.816 \\
\hline A & Lys239 & 184.947 & 0.865 & A & Lys239 & 183.955 & 0.861 & B & Lys239 & 183.575 & 0.859 & $\mathrm{C}$ & Lys239 & 183.431 & 0.858 \\
\hline A & Lys245 & 153.989 & 0.720 & A & Lys 245 & 153.420 & 0.718 & B & Lys 245 & 153.040 & 0.716 & $\mathrm{C}$ & Lys 245 & 153.142 & 0.716 \\
\hline A & Lys 256 & 121.374 & 0.568 & A & Lys 256 & 135.898 & 0.636 & B & Lys 256 & 135.517 & 0.634 & $\mathrm{C}$ & Lys 256 & 136.652 & 0.639 \\
\hline A & Lys273 & 102.949 & 0.482 & A & Lys 273 & 117.257 & 0.549 & B & Lys 273 & 116.855 & 0.547 & $\mathrm{C}$ & Lys 273 & 117.794 & 0.551 \\
\hline A & Lys 285 & 72.402 & 0.339 & A & Lys 285 & 39.866 & 0.187 & B & Lys 285 & 39.701 & 0.186 & $\mathrm{C}$ & Lys 285 & 39.361 & 0.184 \\
\hline A & Lys294 & 145.477 & 0.681 & A & Lys294 & 145.261 & 0.680 & B & Lys294 & 145.962 & 0.683 & $\mathrm{C}$ & Lys294 & 145.570 & 0.681 \\
\hline A & Lys390 & 132.487 & 0.620 & A & Lys390 & 127.896 & 0.598 & B & Lys390 & 126.225 & 0.591 & $\mathrm{C}$ & Lys390 & 126.122 & 0.590 \\
\hline A & Lys393 & 143.664 & 0.672 & A & Lys393 & 156.949 & 0.734 & B & Lys393 & 158.568 & 0.742 & $\mathrm{C}$ & Lys393 & 158.879 & 0.743 \\
\hline A & Lys397 & 154.137 & 0.721 & A & Lys397 & 81.064 & 0.379 & B & Lys397 & 80.455 & 0.376 & $\mathrm{C}$ & Lys397 & 80.574 & 0.377 \\
\hline A & Trp29 & 69.270 & 0.257 & A & Trp29 & 39.003 & 0.145 & B & Trp29 & 38.796 & 0.144 & $\mathrm{C}$ & Trp29 & 38.235 & 0.142 \\
\hline
\end{tabular}


